\section{BICYCLE SADDLES}

IN.JURIES PRODUCED THEREBY AND THEIR RATIONAL REMEDY.

BY JOHN L. SHORT, M.D. ROLLA, MO,

While my association with other cyclists has been limited, it is sufficient, when connected with my medical practice, to enable me to form an opinion as to some of the benefits and ills produced by the wheel. That much benefit is to be derived from a moderate "spin" at the proper hour, for certain patients, is not to be denied. The physical exercise in open air, combined with recreation and pleasure for the mind, is certainly advisable in many cases of neurasthenia, hypochondria, hysteria and other debilities; especially is it to be recommended in many cases of mental derangement. I believe that every insane asylum should be provided with an ample supply of bicycles, and a good track. For all persons who are subject to sedentary life, even though they may seem well, the wheel is not only a source of pleasure and pastime, but nowadays a necessity for health and development of the muscles and nerves.

In order to be a graceful rider, and to promote health, one must sit perfectly erect, with the entire weight on the saddle and pedals. No weight should be on the handlebar. The handlebar need not be touched, except to guide the wheel; and with a good saddle on a good wheel, one can, with a little practice, ride miles and miles without touching the handlebar, making all necessary turns by the motion of the body and feet.

The injuries produced by judicious cycling are but slight. Many good men and women have too hastily condemned the use of the bicycle, claiming that it is a vehicle which carries young girls to prostitution, but my experience teaches me that such theory is not correct. In cycling practiced on the public highways, and at the proper hours, girls may be as safe as in the parlor with closed doors. It is true, some abuse the liberty afforded through cycling; but probably they are already subject to the will of Satan, and the use of the wheel only reveals the fact. Some claim that the irritation of the genitals produced by cycling leads to immoral inclinations, but I am convinced that their conclusions have been drawn more from imagination than from actual knowledge; however, I do not deny that such a result is possible where girls are permitted to ride saddles with high and hard pommels. But when a well-shaped saddle is used, and the rider sits erect, no such effect will occur.

That accidents happen, certain affections are increased, and some diseases are produced, by careless and fast.riding and by riding at improper hours, I am free to admit. But by selecting a clear track, and riding with moderate speed, in daylight, all objectionable features are obviated, and cycling becomes less harmful than riding in the carriage, on cars, or on horseback. I admit that a large percentage of the various saddles breed disease and misery, and should not be used. The hard pommel bruises the soft parts in the pubic arch, and may bring on a train of diseases of the genito-urinary organs of both sexes; and the hard center at the back of the saddle is worse, for it hurts the coccyx when the rider sits erect, causing him to pitch forward, throwing his weight on the handlebar, forming what is called "the bicycle hump." Men and boys prefer to hump forward at the expense of the urethra and other anterior structures, rather than suffer the excruciating pain produced by a bruised coccyx, but women must sit erect, notwithstanding their coccygodynia an affection which no one can fully comprehend unless he has had it.

In order to make my explanation plain, I desire to allude to the anatomic structure of the little triangular extremity of the spinal column, known as the coccyx. In early life this bone is composed of four segments: It is one and a half inches in length, and its proximal piece, which articulates with the sacrum, is one and a half inches wide; but the three last segments are only about one-half inch in width. Thus it is easy to comprehend how this little bone, shaped like a cuckoo's beak, can dip down to the bottom of a crevice which is only a fraction of an inch in depth; especially when the wheel is bumping over stones and railroad tracks. Ossification of the coccyx tak 9 s place as follows: In the first segment, at birth; in the second, at from 5 to 10 years; in the third, from 10 to 15 years; in the fourth, from 15 to 20 years. As age advances, these various pieces unite and form one bone. Later on, in females, the coccyx unites with the sacrum, forming one bone out of what was in youth five pieces. Before ossification of the coccyx takes place, it is cartilaginous and flexible: the sphincter ani is attached to the last segment and draws it forward, while there is nothing to hold it straight, except the delicate extensor coccygis, which is often absent. Consequently, long-continued pressure while the coccyx is soft, will force it against the rectum, thereby lessening the diameter of the pelvis permanently, a point well worth the consideration of obstetricians.

How a slight bruise of the coccyx can produce the severe pain that cyclists suffer is not a mystery when we consider the little ganglion known as the "plexus vasculosum coccygeus," so well supplied with capillary vessels and nerve filaments. It is true that our physiologists have not found a function for this ductless mass called "a gland," but every country wheel. boy who has received a bump on his cuckoo bill is enlightened on the subject, for it resembles a blow on the solar plexus-once felt, never forgotten!

Medical men have not given much attention to the construction of bicycle saddles, but have left the matter to mechanics who know but little of anatomy and physiology. A leading wheelman, who is considered authority on anything concerning a bicycle, recently displayed his lack of anatomic knowledge to me by saying: "When I slip forward, in order to keep from bruising my 'sternum bone' on the back of the saddle, my legs go to sleep from lack of circulation of blood." He meant that in order to save his coccyx, he slips forward till both ischia drop over in front of the saddle, allowing the edges to press into the sacrosciatic notches, interfering with the sciatic nerves. It requires anatomic knowledge to design a perfect saddle, and mechanic art to execute the work as designed.

Of the large variety of saddles heretofore designed, none I have seen are perfect. Many of them have a hard pommel, and bruise the soft parts in front, while all interfere with the coccyx. Several designs of saddles have been advertised in our leading medical journals as perfect; but close observation reveals the fact that the groove in the center is not deep enough, for a groove only the fraction of an inch deep, the bot- 
tom of which is hard, can not furnish perfect protection to a coccyx one and a half inches long. The cut which we see in medical journals, showing the ischia resting on the saddle, while the tip of the coccyx is raised clear above the top of the pubic bones, is incorrect and misleading, for when the spine is erect, the tip of the coccyx comes nearly to a level with the pubic arch. The tuberosities of the ischia and the gluteal muscles should rest firmly on the saddle; but the soft parts in the pubic arch, the sacrosciatic ligaments, and the coccyx, must be free from pressure. The saddle should be too soft to bruise, and too firm to give. The best padding is wool felt. Hair is not good, because it soon becomes displaced, packs down and gets hard. The pneumatic pad is not as good as hair, because it is too hard when well inflated, and, when only partly filled, pressure at one point displaces all the air at that point, allowing the part making the most pressure to come in contact with the hard structure of the saddle. It is also liable to burst, or get punctured.

\section{THE FAUCIAL TONSIL.}

ITS SPHERE AS AN AGENT OF SYSTEMIC INFECTION.

Presented to the Section on Laryngology and Otology, at the Forty-ninth Annual Meeting of the American Medical Association, held at Denver, Colo., June 7-10, 1898.

BY GILLIFORD B. SWEENY, M.D. PITTSBLRG, PA.

The faucial tonsil must be regarded as occupying a very humble position in the human body, either from a histologic or a physiologic standpoint. In fact, the only function of any importance that can be attributed to it is that of leucocytogenesis. Of the entire glandular organism concerned in this work, the tonsil probably occupies the least important part. Its relation to phonation or sexual nutrition need be mentioned only to be dismissed, as every intelligent observer will testify that its absence in no way interferes with the former nor does its presence enhance the latter. $A_{8}$ an agent for the secretion of fluid intended to facili. tate agglutination, it is observed that it is no more active than the mucous membrane which surrounds it and replaces it when removed. The tonsil may be said to be most nearly normal when practically absent from the faucial cayity. It is only when it becomes the seat of morbid phenomena that it requires our serious attention.

It is not the purpose of this paper to dwell at length upon the individual diseases occurring in the tonsil, but rather to refer to them incidentally, especially when considering systemic manifestation having their origin in a morbid state of the tonsil and which in their ultimate results are far-reaching and often most disastrous. We submit for your consideration the following proposition: That the tonsil, while in a state of inflammation, is a frequent and common source of morbid processes which, ultimately, affect the entire human economy. Fortunately the scientific advances of the past few years yield considerable information which goes far toward aiding in establishing the truth of this proposition. We are no longer in doubt as to how the system is primarily attacked in diphtheria. Systemic infection, systemic symptoms must and do invariably follow local morbid processes, which often obtain their hold upon the body through the vulnerability of the organ first attacked. The same may be said of scarlatina, follicular tonsillitis, and, in our judgment, the same occurs in rheumatic fever, in many forms of tuberculosis, and in typhoid fever. That the faucial cavity is a most readily accessible point of attack in infectious diseases is easily understood when we consider the gross anatomic proportions of this region. From its position it may be compared to the smaller and constricted portion of a funnel situated at an abrupt angle. All the agents of respiration and deglutition must come in immediate contact with it in their passage back and forth. However mild the inflammatory process, whether acute or chronic, the tonsil becomes a hotbed for infection, and readily propagates and distributes the morbid products to adjacent and remote organs. Its anatomic construction so closely binds it to the vascular and lymphatic systems, that we are apt to overlook the local and initial manifestation and regard the secondary systematic invasion as the primary morbid process. The immediate result of repeated inflammation of the tonsil, terminating as it does in early life in hyperplasia, or in the mature subject, in true hypertrophy, is familiar to us all. Interfering with nasal respiration it produces the "mouth breather," with its train of deleterious results. Improperly filtered, moistened and warm air is hastily conveyed to the lungs, oxygenation is seriously interfered with, which promptly displays its ill effects in the dull, listless eye, impaired mentality, the pallid complexion, the contracted chest, and the anemic and often wasted muscular organism. Hypostatic congestion in the nasal tract is followed by interference with the normal functions of the organs of sight, smell and hearing, and encourages the hypertrophic process to extend indefinitely in all directions. None can deny the statement that the tonsil offers special facilities for the propagation of the diphtheritic bacillus; failing to admit this, how can we account for the fact that the vast majority of cases originate here? We can not generalize and say that it is because of the susceptibility of mucous tissues, for how really few cases originate in the nostril, the eye, or other equally accessible points.

I am fully convinced that not only is the tonsil a vulnerable point for diphtheria, but that when in a morbid state it becomes often a culture-medium for the propagation of germs which otherwise would perish before gaining access to the body of their host. The same is true to even a greater degree in those two other highly contagious and infectious diseasesscarlatina and follicular tonsillitis. While diphtheria may primarily attack some other organ than the tonsil. scarlatina certainly very seldom, and infectious follicular tonsillitis never, selects any other organ as a point of initial invasion. We are now able, I believe, to establish almost beyond doubt the infectious nature of rheumatism. One can not fail to observe the analogy in the systemic symptoms of follicular tonsillitis and acute rheumatism. While we have not yet been able to isolate a germ strictly characteristic of rheumatism, yet in this disease we find in the blood streptococci and staphylococci, which characterize follicular tonsillitis and are found in the tonsil when affected in that disease.

The metabolic theory as to the causation of rheumatism is certainly barren of the essential facts necessary to establish even its plausibility. To assume that defective assimilation under widely varying circumstances may produce a specific chemic agent- 